\title{
Summary I - Accelerator Ion sources, Fundamentals and Diagnostics
}

\author{
Douglas P. Moehs \\ Fermilab, Accelerator Division, P.O. Box 500 Batavia IL, 60561 USA
}

\begin{abstract}
The $11^{\text {th }}$ International Symposium on the Production and Neutralization of Negative Ions and Beams was held in Santa Fe, New Mexico on September 12-15, 2006 and was hosted by Los Alamos National Laboratory. This summary covers the first three oral sessions of the symposium.
\end{abstract}

Keywords: Accelerator, Ion Source, Negative Ions, Hydrogen, Laser Applications, Computer Modeling

PACS: 29.17.+w, 41.75.Cn, 07.77.Ka, 42.62.-b, 52.65.-y

\section{GENERAL COMMENTS}

The Eleventh International Symposium on the Production and Neutralization of Negative Ions and Beams (PNNIB) was held in Santa Fe, New Mexico on September 12-15, 2006. The symposium continues to draw many participants from both the accelerator and fusion based communities as well as a few participants from industry. The oral sessions at this symposium consisted of 37 contributions presented by participants from 11 different countries. Due to Visa problems Dr. Belchenko was unable to attend, however his abstract was read by proxy and his paper appears within these proceedings. Dr. Rosen opened the symposium with an overview of the conception and construction of LANSCE.

A host of fruitful ideas and collaborations have sprung out of the PNNIB workshops crossing communities and ion source types and for this the conference attendees thank our International Organizing Committee. We are also grateful to LANL and the local organizing committee for hosting the conference. The venue was excellent and the meals left us satisfied. We would especially like to thank Roderich Keller, Gary Rouleau, Angelina Gurulé-Sanchez, Linda Chavez, Theresa Chavez and Lisa Padilla for their efforts in making our conference experience a pleasant one.

\section{SUMMARIES}

\section{Accelerator Ion Sources}

Table 1 lists some of the front-end beam specifications of eight potentially new or upgraded machines, being considered around the world, in comparison to the DESY 
and BNL front-ends. From Table 1 it is clear that there is a trend toward higher average currents and longer pulse lengths without sacrificing emittance. At the SNS the need is immanent and thus the ion source R\&D being carried out there is leading the charge toward meeting these requirements, and of course many eyes are watching with interest. LEBT (low energy beam transport) energies are also notably increased for these new machines leading in some cases to multi-acceleration gap designs. This is particularly notable at the CERN-SPL where the $\mathrm{H}^{-}$ions will pass through a

TABLE 1: Front end beam parameters for several proposed machines in comparison to existing machines at DESY in Germany and at BNL in the United States.

\begin{tabular}{|l|l|l|l|l|l|}
\hline Laboratory & $\begin{array}{l}\text { Current } \\
(\mathrm{mA})\end{array}$ & $\begin{array}{l}\text { Emittance } \\
\text { pi-mm-mr } \\
(\mathrm{nor} ., \mathrm{rms})\end{array}$ & $\begin{array}{l}\text { Pulse } \\
\text { Length } \\
(\mathrm{ms})\end{array}$ & $\begin{array}{l}\text { Rep. } \\
\text { Rate } \\
(\mathrm{Hz})\end{array}$ & $\begin{array}{l}\text { LEBT } \\
\text { Energy } \\
(\mathrm{keV})\end{array}$ \\
\hline $\begin{array}{l}\text { DESY (multi cusp RF) } \\
\text { BNL (magnetron) }\end{array}$ & $\begin{array}{l}40 \\
50\end{array}$ & $\begin{array}{l}0.25 \\
0.4\end{array}$ & $\begin{array}{l}0.15 \\
0.7\end{array}$ & $\begin{array}{l}8 \\
6\end{array}$ & $\begin{array}{l}35 \\
35\end{array}$ \\
\hline SNS upgrade & $50 / 100$ & $0.2 / \leq 0.35$ & 1 & 60 & 65 \\
\hline $\begin{array}{l}\text { CERN-SPL } \\
\text { Phase A / B }\end{array}$ & $40 / 80$ & 0.25 & $0.4 / 0.7$ & $2 / 50$ & 95 \\
\hline $\begin{array}{l}\text { Fermilab-HINS } \\
\text { Initial / Final }\end{array}$ & $13 / 40$ & 0.24 & $3 / 1$ & $2.5 / 10$ & 50 \\
\hline $\begin{array}{l}\text { BSNS* } \\
\text { Beijing, China }\end{array}$ & 40 & 0.2 & 0.2 & 25 & 75 \\
\hline ISIS upgrade & 70 & 0.17 & 1.5 & 50 & 70 \\
\hline
\end{tabular}

secondary acceleration gap of $60 \mathrm{kV}$ [Th. Meinschad]. Every major ion source type is being considered for improvement. However, the external-antenna RF (radio frequency) driven multi-cusp source appears to be in vogue at the present time, offering the potential of increased duty factor (DF) and $\mathrm{H}^{-}$currents without giving up source lifetime or leading to excessive emittance growth. Data, in particular for emittance, is still too sparse to state these claims as fact.

J. Peters reported that a collaborative effort between DESY, SNS, Fermilab and CERN made possible $40 \mathrm{~mA}(3 \mathrm{~ms})$ pulses at $0.5 \mathrm{~Hz}$ on the DESY RF source. No cesium is added to this system. A variant of this source will be used on the CERNSPL project [Th. Meinschad]. On the SNS RF source, many changes and improvements are being tested [R. Welton]. Under this aggressive R\&D effort, an external antenna source produced a current of $80 \mathrm{~mA}(0.2 \mathrm{~ms})$; on the internal antenna source a hollow anode plasma gun is now used to ignite the plasma and an elemental cesium oven has improved stability of the source producing $60 \mathrm{~mA}(1.2 \mathrm{~ms})$. Investigation of a higher gradient extraction system also appears to be a promising path toward higher current. For the SNS upgrade project a new magnetic LEBT is being design including two ion sources to improve source availability [M. Stockli].

Work on surface plasma sources of the Penning and Magnetron variety is also continuing at ISIS and Fermilab respectively. At ISIS the Penning source output has been increased to $70 \mathrm{~mA}(1.5 \mathrm{~ms})$ and effort is being made to understand why the 
emittance is larger than expected [D. Faircloth]. Work done in collaboration with Oxford University showed that the beam energy spread is $17.6 \mathrm{eV} \pm 1.5 \mathrm{eV}$ and thus is not contributing to the large emittances. A hollow-cathode Penning source is being developed at the Budker Institute for DC beams and it has produced $\mathrm{H}^{-}$currents up to $15 \mathrm{~mA}$ (DC) [Y. Belchenko]. The Magnetron pulse length at BNL was briefly tested out to $1.6 \mathrm{~ms}$ with a peak current of $60 \mathrm{~mA}$ [D. Moehs]. In addition, an emittance measurement at Fermilab for a beam current of $11 \mathrm{~mA}$ was in good agreement with extrapolated historically published Magnetron emittance data. This work indicates that a Magnetron should be able to meet the first-phase HINS (High Intensity Neutrino Source) $\mathrm{H}^{-}$beam requirements, see Table 1 .

Polarized $\mathrm{H}^{-}$beam intensities continued to be improved at COSY [R. Gebel]. In 2005 they achieved a beam current of 50 micro-amps $(20 \mathrm{~ms})$ operating at $0.5 \mathrm{~Hz}$, a factor of 3 better than previous experimental runs.

Development of ECR-driven sources for the production of $\mathrm{H}^{-}$beams continues to be challenging. At CEA Saclay [O. Tuske] a $2.45 \mathrm{GHz}$ ECR test stand has produced a maximum of $3.8 \mathrm{~mA}(10 \mathrm{~ms})$ at $10 \mathrm{~Hz}$ without cesium. Work is underway to add an octuple multi-cusp field to this source. R. Keller reported on the HYBRIS source work at LBNL, and concluded that the electron density is simply not high enough to produce significant beam currents. This effort, to couple a plasma driver with a magnetically divided multi-cusp chamber, has lead to collaborative efforts to utilize a helicon plasma generator as the plasma cathode, which can produce higher electron densities, $\mathrm{n}_{\mathrm{e}}=10^{12}-10^{14}$ per $\mathrm{cm}^{3}$, as compared to ECR driven plasmas [R. Welton, $\mathrm{O}$. Tarvainen, P. Farrell].

Optimizing $\mathrm{H}^{-}$production in cesiated multi-cusp sources was discussed in detail particularly in regard to the magnetic separation of the drive plasma and production regions. In a Reflective Discharge Source the relative $\mathrm{H}^{-}$contribution from surface production was shown to dominate over volume production [V. Goretsky]. On the other hand, Cs enhancement effects in the production process of highly vibrational states are still being considered theoretically [S. Longo].

Two novel source concepts were also presented. R. Becker proposed that a source producing 30-50 eV protons could be used as the driver for a multi-cusp source based on the fact that the relative cross-sections for producing highly vibrational states of $\mathrm{H}_{2}$ with protons is 2 orders of magnitude higher than for electrons. Andrew Holmes showed that the use of magnetic fields, while aiding in the production of $\mathrm{H}^{-}$by separating hot and cold electrons, contributes to the emittance and thus the divergence of the ion beam due to plasma non-uniformities resulting from a $\mathrm{J} \times \mathrm{B}$ drift which distorts the beam-plasma meniscus. O. Fukumasa has also come to this conclusion and has constructed a plasma test system utilizing a biased grid to separate hot and cold electrons. Data from this system indicates that the electron density and temperatures are similar for magnetic and electrostatic filter fields.

Accelerator - Comments

Figure 1 shows a scatter plot of emittance and brightness as a function of current for a selection of accelerator based ion sources. From the JAERI datum point one might argue that the best research path toward a long lived high performance source is to invest more time in filament R\&D. Among the other accelerator based ion $\mathrm{H}^{-}$ 
sources there does not appear to be an outstanding performer with respect to emittance. It may be that Andrew Holmes' meniscus distortion theories play a part in this based on the fact that every operational ion source uses a transverse magnetic field to reduce the $\mathrm{H}^{-} / \mathrm{e}$ extraction ratio, improve the plasma confinement and, in the case of multi-cusp volume sources, to improve the electron energy separation.

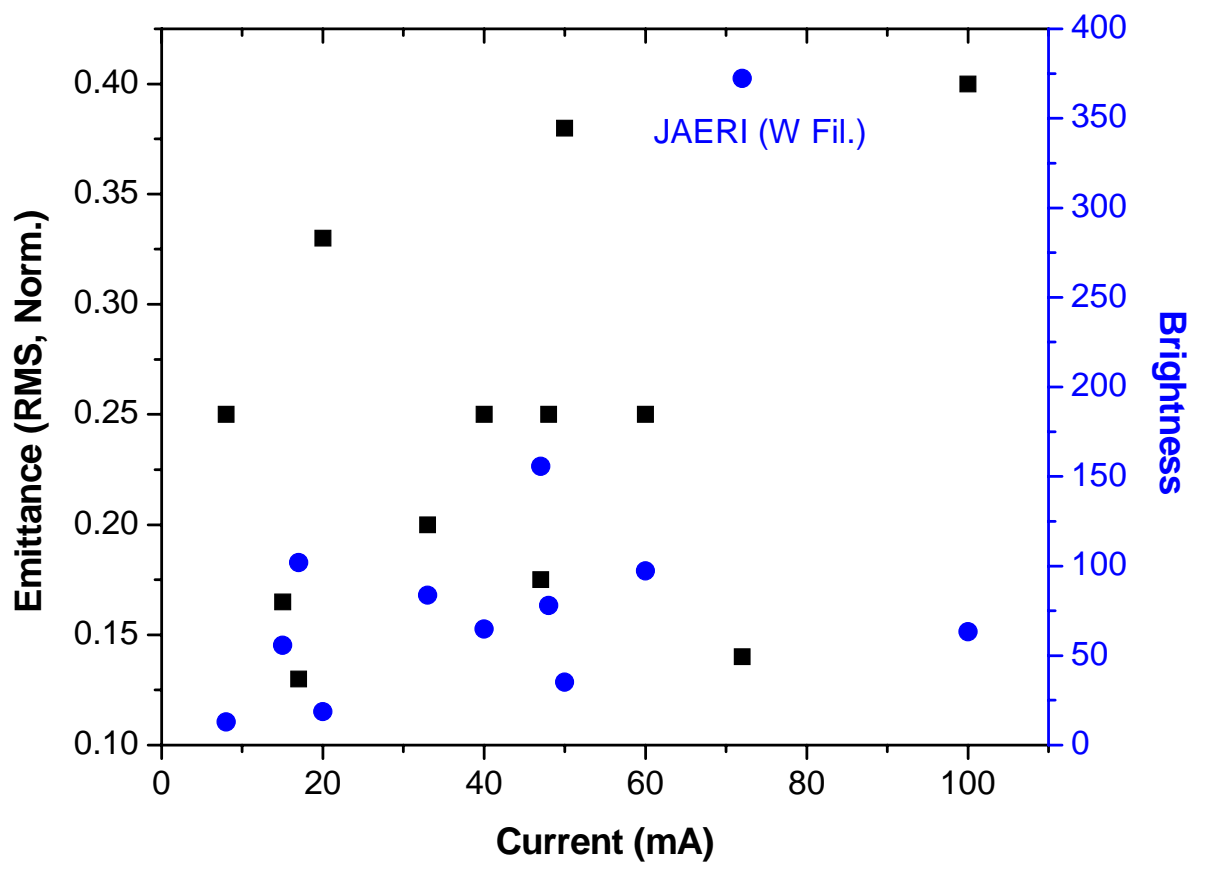

FIGURE 1. Emittance and brightness as a function of current for a representative selection of accelerator based ion sources.

\section{Fundamentals}

Particle in cell (PIC) simulations coupled with Monte Carlo (MC) fitting of the available data has become the norm for understanding $\mathrm{H}^{-}$production and extraction properties from plasma based ion sources. These codes have been adapted to include detailed particle collision processes of multiple species, plasma-surface and plasmabeam processes, external electric and magnetic fields, space-charge, thermal effects and chemical kinetics. At this workshop, applications of MC-PIC simulations to aspects of large volume sources and the LANL Surface Converter Source were presented.

For the JAEA 10 Ampere negative ion source the $\mathrm{H}_{0}$ atom production rate and spatial distributions were calculated as a function of electron energy for the cesiated case [N. Takado]. This study showed that highly energetic electrons contributed significantly to $\mathrm{H}_{0}$ production. On the IPP RF negative ion source, charged and neutral particle motion in $1 \mathrm{D}(\mathrm{z})$ were studied in the $\mathrm{H}^{-}$production and extraction regions [F. Taccogna]. $\mathrm{H}^{-}$survival probabilities in these regions were also presented [D. Wunderlich]. These two studies focused on the influence of the magnetic filter field and plasma grid bias. Two lines along the z-axis, one ending on the plasma grid 
and one through the extraction aperture, were used to contract surface interaction and non-interaction paths.

The group at Bari continues to push the hybrid kinetic model they developed to include simulations of non equilibrium plasmas [S. Longo]. Effects of space charge, state to state vibrational $\mathrm{H}_{2}$ populations, and cesiation of the walls were presented. The Bari group is also working on numerically extending the QCT (quasi-classical trajectory) state-to-state cross sections for $\mathrm{D}-\mathrm{D}_{2}$ and $\mathrm{T}-\mathrm{T}_{2}$ by scaling $\mathrm{H}-\mathrm{H}_{2}$ cross sections as well as deriving the resonant dissociative attachment and vibrationally excitation cross sections for the 14-eV resonance [F. Esposito].

For the LANL Surface Converter Source a $2 \mathrm{D}(\mathrm{r}, \mathrm{z})-3 \mathrm{D}\left(\mathrm{v}_{\mathrm{r}}, \mathrm{v}_{\mathrm{z}}, \mathrm{v}_{\varphi}\right)$ code has been developed [E. Chacon-Gocher]. This MC-PIC code models the entire ion source from the cathode surface through the plasma meniscus into the extractor electrode and it is the first code to really push toward full $3 \mathrm{D} \mathrm{H}^{-}$plasma simulations. Further efforts to produce 3D codes should be high priority because of the enormous potential these codes have to unlock the complex physics of $\mathrm{H}^{-}$ion sources. Collaborative efforts, already forming, in MC-PIC simulations will accelerate this work.

\section{Diagnostics}

In each of the three diagnostic presentations photo-detachment of $\mathrm{H}^{-}$ions played an important role. As with RF ion sources, laser diagnostics appear to be in vogue, offering a relatively non-destructive way of measuring beam profiles, emittances and $\mathrm{H}^{-}$densities. This is particularly important for high powered machines where thermal stresses and component activation need to be considered. At Rutherford Appleton Laboratory laser based diagnostics are being developed for the $60 \mathrm{~mA}, 3 \mathrm{MeV}$ Front End Test Stand [C. Gabor]. As part of this work, a novel technique for obtaining 2D transverse beam profiles at $70 \mathrm{keV}$ is being developed by measuring the particle density versus laser angle and position as the laser beam is rotated through the ion beam.

For large fusion ion sources knowledge of electron energy distribution functions (EEDF) are important for improving radiative-collision models and MC-PIC simulations [B. Crowley]. To assess the impact of non-Maxwelian EEDF's on rate coefficients for specific $\mathrm{H}^{-}$production and destruction processes Langmuir probe measurements have been carried on the KAMABOKO III source at Cadarache.

Tests of an External Cavity Ring Down technique for measuring linear-integrated $\mathrm{H}^{-}$densities are being carried out on the KAMABOKO III ion source [R. Faulkner]. This novel technique is achieved by utilizing polarized light to form the optical ring down cavity. This method increases detectable light levels and improves statistics but requires sophisticated detection electronics and data analysis techniques. 